\title{
The Photoevaporation of Dwarf Galaxies during Reionization
}

\section{Citation}

Barkana, Rennan, and Abraham Loeb. 1999. “The Photoevaporation of Dwarf Galaxies during Reionization." The Astrophysical Journal 523 (1): 54-65. https://doi.org/10.1086/307724.

\section{Permanent link}

http://nrs.harvard.edu/urn-3:HUL.InstRepos:41393254

\section{Terms of Use}

This article was downloaded from Harvard University's DASH repository, and is made available under the terms and conditions applicable to Other Posted Material, as set forth at http:// nrs.harvard.edu/urn-3:HUL.InstRepos:dash.current.terms-of-use\#LAA

\section{Share Your Story}

The Harvard community has made this article openly available.

Please share how this access benefits you. Submit a story.

\section{Accessibility}


The AstrophysiCAL JouRnAL, 523:54-65, 1999 September 20

(C) 1999. The American Astronomical Society. All rights reserved. Printed in U.S.A.

\title{
THE PHOTOEVAPORATION OF DWARF GALAXIES DURING REIONIZATION
}

\author{
RENNAN BARKANA \\ Institute for Advanced Study, Olden Lane, Princeton, NJ 08540; barkana@ias.edu \\ AND \\ ABRAHAM LOEB \\ Astronomy Department, Harvard University, 60 Garden Street, Cambridge, MA 02138; aloeb@cfa.harvard.edu \\ Received 1999 January 12; accepted 1999 May 5
}

\begin{abstract}
During the period of reionization, the universe was filled with a cosmological background of ionizing radiation. By that time a significant fraction of the cosmic gas had already been incorporated into collapsed galactic halos with virial temperatures $\lesssim 10^{4} \mathrm{~K}$ that were unable to cool efficiently. We show that photoionization of this gas by the fresh cosmic UV background boiled the gas out of the gravitational potential wells of its host halos. We calculate the photoionization heating of gas inside spherically symmetric dark matter halos and assume that gas that is heated above its virial temperature is expelled. In popular cold dark matter models, the Press-Schechter halo abundance implies that $\sim 50 \%-90 \%$ of the collapsed gas was evaporated at reionization. The gas originated from halos below a threshold circular velocity of $\sim 10-15 \mathrm{~km} \mathrm{~s}^{-1}$. The resulting outflows from the dwarf galaxy population at redshifts $z=5-10$ affected the metallicity and the thermal and hydrodynamic states of the surrounding intergalactic medium. Our results suggest that stellar systems with a velocity dispersion $\lesssim 10 \mathrm{~km} \mathrm{~s}^{-1}$, such as globular clusters or the dwarf spheroidal galaxies of the Local Group, did not form directly through cosmological collapse at high redshifts.
\end{abstract}

Subject headings: cosmology: theory — galaxies: formation — galaxies: halos — radiative transfer

\section{INTRODUCTION}

The formation of galaxies is one of the most important, yet unsolved, problems in cosmology. The properties of galactic dark matter halos are shaped by gravity alone and have been rigorously parameterized in hierarchical cold dark matter (CDM) cosmologies (e.g., Navarro, Frenk, \& White 1997, hereafter NFW). However, the complex processes involving gas dynamics, chemistry and ionization, and cooling and heating, which are responsible for the formation of stars from the baryons inside these halos, have still not been fully explored theoretically.

Recent theoretical investigations of early structure formation in CDM models have led to a plausible picture of how the formation of the first cosmic structures leads to reionization of the intergalactic medium (IGM). The bottom-up hierarchy of CDM cosmologies implies that the first gaseous objects to form in the universe have a low mass, just above the cosmological Jeans mass of $\sim 10^{4} M_{\odot}$ (see, e.g., Haiman, Thoul, \& Loeb 1996b and references therein). The virial temperature of these gas clouds is only a few hundred $\mathrm{K}$, and so their metal-poor primordial gas can cool only as a result of the formation of molecular hydrogen, $\mathrm{H}_{2}$. However, $\mathrm{H}_{2}$ molecules are fragile and were easily photodissociated throughout the universe by trace amounts of starlight (Stecher \& Williams 1967; Haiman, Rees, \& Loeb 1996a) that were well below the level required for complete reionization of the IGM. Following the prompt destruction of their molecular hydrogen, the early low-mass objects maintained virialized gaseous halos that were unable to cool or fragment into stars. Most of the stars responsible for the reionization of the universe formed in more massive galaxies, with virial temperatures $T_{\mathrm{vir}} \gtrsim 10^{4} \mathrm{~K}$, where cooling due to atomic transitions was possible. The corresponding mass of these objects at $z \sim 10$ was $\sim 10^{8} M_{\odot}$, typical of dwarf galaxies.
The lack of a Gunn-Peterson trough and the detection of Ly $\alpha$ emission lines from sources out to redshifts $z=5.6$ (Weymann et al. 1998; Dey et al. 1998; Spinrad et al. 1998; $\mathrm{Hu}$, Cowie, \& McMahon 1998) demonstrates that reionization due to the first generation of sources must have occurred at yet higher redshifts; otherwise, the damping wing of Ly $\alpha$ absorption by the neutral IGM would have eliminated the Ly $\alpha$ line in the observed spectrum of these sources (Miralda-Escudé 1998). Popular CDM models predict that most of the intergalactic hydrogen was ionized at a redshift $8 \lesssim z \lesssim 15$ (Gnedin \& Ostriker 1997; Haiman $\&$ Loeb 1998, 1998c). The end of the reionization phase transition resulted in the emergence of an intense UV background that filled the universe and heated the IGM to temperatures of $\sim 1-2 \times 10^{4} \mathrm{~K}$ (Haiman \& Loeb 1999; Miralda-Escudé, Haehnelt, \& Rees 1999). After ionizing the rarefied IGM in the voids and filaments on large scales, the cosmic UV background penetrated the denser regions associated with the virialized gaseous halos of the first generation of objects. Since a major fraction of the collapsed gas had been incorporated by that time into halos with a virial temperature $\lesssim 10^{4} \mathrm{~K}$, photoionization heating by the cosmic UV background could have evaporated much of this gas back into the IGM. No such feedback was possible at earlier times, since the formation of internal UV sources was suppressed by the lack of efficient cooling inside most of these objects.

The gas reservoir of dwarf galaxies with virial temperatures $\lesssim 10^{4} \mathrm{~K}$ (or equivalently a one-dimensional velocity dispersion $\lesssim 10 \mathrm{~km} \mathrm{~s}^{-1}$ ) could not be immediately replenished. The suppression of dwarf galaxy formation at $z>2$ has been investigated both analytically (Rees 1986; Efstathiou 1992) and with numerical simulations (Thoul \& Weinberg 1996; Quinn, Katz, \& Efstathiou 1996; Weinberg, Hernquist, \& Katz 1997; Navarro \& Steinmetz 1997). 
The dwarf galaxies that were prevented from forming after reionization could have eventually collected gas at $z=1-2$, when the UV background flux declined sufficiently (Babul \& Rees 1992; Kepner, Babul, \& Spergel 1997). The reverse process during the much earlier reionization epoch has not been addressed in the literature. (However, note that the photoevaporation of gaseous halos was considered by Bond, Szalay, \& Silk (1988) as a model for Ly $\alpha$ absorbers at lower redshifts $z \sim 4$.)

In this paper we focus on the reverse process by which gas that had already settled into virialized halos by the time of reionization was evaporated back into the IGM because of the cosmic UV background that emerged first at that epoch. The basic ingredients of our model are presented in $\S 2$. In order to ascertain the importance of a self-shielded gas core, we include a realistic, centrally concentrated dark halo profile and also incorporate radiative transfer. Generally we find that self-shielding has a small effect on the total amount of evaporated gas, since only a minor fraction of the gas halo is contained within the central core. Our numerical results are described in $\S 3$. In particular, we show the conditions in the highest mass halo that can be disrupted at reionization. We also use the Press \& Schechter (1974) prescription for halo abundance to calculate the fraction of gas in the universe that undergoes the process of photoevaporation. Our versatile semianalytic approach has the advantage of being able to yield the dependence of the results on a wide range of reionization histories and cosmological parameters. Clearly, the final state of the gas halo depends on its dynamical evolution during its photoevaporation. We adopt a rough criterion for the evaporation of gas based on its initial interaction with the ionizing background. The precision of our results could be tested in specific cases by future numerical simulations. In $\S 4$ we discuss the potential implications of our results for the state of the IGM and for the early history of low-mass galaxies in the local universe. Finally, we summarize our main conclusions in $\S 5$.

\section{A MODEL FOR HALOS AT REIONIZATION}

We consider gas situated in a virialized dark matter halo. We adopt the prescription for obtaining the density profiles of dark matter halos at various redshifts from the Appendix of NFW, modified to include the variation of the collapse overdensity $\Delta_{c}$. Thus, a halo of mass $M$ at redshift $z$ is characterized by a virial radius,

$$
\begin{aligned}
r_{\mathrm{vir}}= & 0.756\left(\frac{M}{10^{8} h^{-1} M_{\odot}}\right)^{1 / 3}\left[\frac{\Omega_{0}}{\Omega(z)} \frac{\Delta_{c}}{200}\right]^{-1 / 3} \\
& \times\left(\frac{1+z}{10}\right)^{-1} h^{-1} \mathrm{kpc},
\end{aligned}
$$

or a corresponding circular velocity,

$$
\begin{aligned}
V_{c} & =\left(\frac{G M}{r_{\mathrm{vir}}}\right)^{1 / 2} \\
& =31.6\left(\frac{r_{\mathrm{vir}}}{h^{-1} \mathrm{kpc}}\right)\left[\frac{\Omega_{0}}{\Omega(z)} \frac{\Delta_{c}}{200}\right]^{1 / 2}\left(\frac{1+z}{10}\right)^{3 / 2} \mathrm{~km} \mathrm{~s}^{-1} .
\end{aligned}
$$

The density profile of the halo is given by

$$
\rho(r)=\frac{3 H_{0}^{2}}{8 \pi G}(1+z)^{3} \frac{\Omega_{0}}{\Omega(z)} \frac{\delta_{c}}{c x(1+c x)^{2}},
$$

where $x=r / r_{\text {vir }}$ and $c$ depends on $\delta_{c}$ for a given mass $M$. We include the dependence of halo profiles on $\Omega_{0}$ and $\Omega_{\Lambda}$, the current contributions to $\Omega$ from nonrelativistic matter and a cosmological constant, respectively (see Appendix A for complete details).

Although the NFW profile provides a good approximation to halo profiles, there are indications that halos may actually develop a core (e.g., Burkert 1995; Kravtsov et al. 1998; see, however, Moore et al. 1998). In order to examine the sensitivity of the results to model assumptions, we consider several different gas and dark matter profiles, keeping the total gas fraction in the halo equal to the cosmological baryon fraction. The simplest case we consider is an equal NFW profile for the gas and the dark matter. In order to include a core, instead of the NFW profile of equation (3), we also consider the density profile of the form fit by Burkert (1995) to dwarf galaxies,

$$
\rho(r)=\frac{3 H_{0}^{2}}{8 \pi G}(1+z)^{3} \frac{\Omega_{0}}{\Omega(z)} \frac{\delta_{c}}{(1+b x)\left[1+(b x)^{2}\right]},
$$

where $b$ is the inverse core radius, and we set $\delta_{c}$ by requiring the mean overdensity to equal the appropriate value, $\Delta_{c}$, in each cosmology (see Appendix A). We also consider two cases where the dark matter follows an NFW profile but the gas is in hydrostatic equilibrium with its density profile determined by its temperature distribution. In one case, we assume the gas is isothermal at the halo virial temperature, given by

$$
\begin{aligned}
T_{\mathrm{vir}} & =\frac{\mu V_{c}^{2}}{2 k_{B}} \\
& =36,100 \frac{\mu}{0.6 m_{p}}\left(\frac{r_{\mathrm{vir}}}{h^{-1} \mathrm{kpc}}\right)^{2} \frac{\Omega_{0}}{\Omega(z)} \frac{\Delta_{c}}{200}\left(\frac{1+z}{10}\right)^{3} \mathrm{~K},
\end{aligned}
$$

where $\mu$ is the mean molecular weight as determined by ionization equilibrium, and $m_{p}$ is the proton mass. The spherical collapse simulations of Haiman et al. (1996b) find a postshock gas temperature of roughly twice the value given by equation (5), so we also compare with the result of setting $T=2 T_{\mathrm{vir}}$. In the second case, we let the gas cool for a time equal to the Hubble time at the redshift of interest, $z$. Gas above $10^{4} \mathrm{~K}$ cools rapidly due to atomic cooling until it reaches a temperature near $10^{4} \mathrm{~K}$, where the cooling time rapidly diverges. In this case, hydrostatic equilibrium yields a highly compact gas cloud when the halo virial temperature is greater than $10^{4} \mathrm{~K}$. In reality, of course, a fraction of the gas may fragment and form stars in these halos. However, this caveat hardly affects our results since only a small fraction of the gas which evaporates is contained in halos with $T_{\text {vir }}>10^{4} \mathrm{~K}$. Throughout most of our subsequent discussion we consider the simple case of identical NFW profiles for both the dark matter and the gas, unless indicated otherwise.

We assume a helium mass fraction of $Y=0.24$, and include it in the calculation of the ionization equilibrium state of the gas as well as its cooling and heating (see, e.g., Katz, Weinberg, \& Hernquist 1996). We adopt the various reaction and cooling rates from the literature, including the rates for collisional excitation and dielectronic recombination from Black (1981); the recombination rates from Verner \& Ferland (1996), and the recombination cooling rates from Ferland et al. (1992) with a fitting formula by J. Miralda-Escudé (1998, private communication). Collisional 
ionization rates are adopted from Voronov (1997), with the corresponding cooling rate for each atomic species given by its ionization rate multiplied by its ionization potential. We also include cooling by Bremsstrahlung emission with a Gaunt factor from Spitzer \& Hart (1971) and by Compton scattering off the microwave background (e.g., Shapiro \& Kang 1987).

In assessing the effect of reionization, we assume for simplicity a sudden turn-on of an external radiation field with a specific intensity per unit frequency, $v$ :

$$
I_{v, 0}=10^{-21} I_{21}(z)\left(v / v_{\mathrm{L}}\right)^{-\alpha} \operatorname{ergs~} \mathrm{cm}^{-2} \mathrm{~s}^{-1} \mathrm{sr}^{-1} \mathrm{~Hz}^{-1},
$$

where $v_{\mathrm{L}}$ is the Lyman limit frequency. Our treatment of the response of the cloud to this radiation, as outlined below, is not expected to yield different results with a more gradual increase of the intensity with cosmic time. The external intensity $I_{21}(z)$ is responsible for the reionization of the IGM, and so we normalize it to have a fixed number of ionizing photons per baryon in the universe. We define the ionizing photon density as

$$
n_{\gamma}=\int_{v_{\mathrm{L}}}^{\infty} \frac{4 \pi I_{v, 0}}{h v c} \frac{\sigma_{\mathrm{HI}}(v)}{\sigma_{\mathrm{HI}}\left(v_{\mathrm{L}}\right)} d v,
$$

where the photoionization efficiency is weighted by the photoionization cross section of $\mathrm{H} \mathrm{I}, \sigma_{\mathrm{HI}}(v)$, above the Lyman limit. The mean baryon number density is

$$
n_{b}=2.25 \times 10^{-4}\left(\frac{1+z}{10}\right)^{3}\left(\frac{\Omega_{b} h^{2}}{0.02}\right) \mathrm{cm}^{-1} .
$$

Throughout the paper we refer to proper densities rather than comoving densities. As our standard case we assume a post-reionization ratio of $n_{\gamma} / n_{b}=1$, but we also consider the effect of setting $n_{\gamma} / n_{b}=0.1$. For example, $\alpha=1.8$ and $n_{\gamma} / n_{b}=1$ yield $I_{21}=1.0$ at $z=3$ and $I_{21}=3.5$ at $z=5$, close to the values required to satisfy the Gunn-Peterson constraint at these redshifts (see, e.g., Efstathiou 1992). Note that $n_{\gamma} / n_{b} \gtrsim 1$ is required for the initial ionization of the gas in the universe (although this ratio may decline after reionization).

We assume that the above uniform UV background illuminates the outer surface of the gas cloud, located at the virial radius $r_{\mathrm{vir}}$, and penetrates from there into the cloud. The radiation photoionizes and heats the gas at each radius to its equilibrium temperature, determined by equating the heating and cooling rates. The latter assumption is justified by the fact that both the recombination time and the heating time are initially shorter than the dynamical time throughout the halo. At the outskirts of the halo the dynamics may start to change before the gas can be heated up to its equilibrium temperature, but this simply means that the gas starts expanding out of the halo during the process of photoheating. This outflow should not alter the overall fraction of evaporated gas.

The process of reionization is expected to be highly nonuniform because of the clustering of the ionizing sources and the clumpiness of the IGM. As time progresses, the H II regions around the ionizing sources overlap, and each halo is exposed to ionizing radiation from an ever increasing number of sources. While the external ionizing radiation may at first be dominated by a small number of sources, it quickly becomes more isotropic as its intensity builds up with time (e.g., Haiman \& Loeb 1998, 1999; MiraldaEscudé et al. 1999). The evolution of this process depends on the characteristic clustering scale of ionizing sources and their correlation with the inhomogeneities of the IGM. In particular, the process takes more time if the sources are typically embedded in dense regions of the neutral IGM that need to be ionized first before their radiation shines on the rest of the IGM. However, in our analysis we do not need to consider these complications, since the total fraction of evaporated gas in bound halos depends primarily on the maximum intensity achieved at the end of the reionization epoch.

In computing the effect of the background radiation, we include self-shielding of the gas, which is important at the high densities obtained in the core of high-redshift halos. For this purpose, we include radiative transfer through the halo gas and photoionization by the resulting anisotropic radiation field in the calculation of the ionization equilibrium. We also include the fact that the ionizing spectrum becomes harder at inner radii, since the outer gas layers preferentially block photons with energies just above the Lyman limit. We neglect self-shielding due to helium atoms. Appendix B summarizes our simplified treatment of the radiative transfer equations.

Once the gas is heated throughout the halo, some fraction of it acquires a sufficiently high temperature that it becomes unbound. This gas expands due to the resulting pressure gradient and eventually evaporates back to the IGM. The pressure gradient force (per unit volume) $k_{B} \nabla(T \rho / \mu)$ competes with the gravitational force of $\rho G M / r^{2}$. Because of the density gradient, the ratio between the pressure force and the gravitational force is roughly the ratio between the thermal energy $\sim k_{B} T$ and the gravitational binding energy $\sim \mu G M / r$ (which is $\sim k_{B} T_{\text {vir }}$ at $r_{\mathrm{vir}}$ ) per particle. Thus, if the kinetic energy exceeds the potential energy (or roughly if $T>T_{\mathrm{vir}}$ ), the repulsive pressure gradient force exceeds the attractive gravitational force and expels the gas on a dynamical time (or faster for halos with $\left.T \gg T_{\text {vir }}\right)$.

We compare the thermal and gravitational energy (both of which are functions of radius) as a benchmark for deciding which gas shells are expelled from each halo. Note that the infall of fresh IGM gas into the halo is also suppressed because of its excessive gas pressure, produced by the same photoionization heating process.

This situation stands in contrast to feedback due to supernovae, which depends on the efficiency of converting the mechanical energy of the supernovae into thermal energy of the halo gas. The ability of supernovae to disrupt their host dwarf galaxies has been explored in a number of theoretical papers (e.g., Larson 1974; Dekel \& Silk 1986; Vader 1986, 1987). However, numerical simulations (Mac Low \& Ferrara 1999) find that supernovae produce a hole in the gas distribution through which they expel the shockheated gas, leaving most of the cooler gas still bound. In the case of reionization, on the other hand, energy is imparted to the gas directly by the ionizing photons. A halo for which a large fraction of the gas is unbound by reionization is thus prevented from further collapse and star formation.

When the gas in each halo is initially ionized, an ionization shock front may be generated (see the discussion of Ly $\alpha$ absorbers by Donahue \& Shull 1987). The dynamics of such a shock front have been investigated in the context of the interstellar medium by Bertoldi \& McKee (1990) and Ber- 
toldi (1989). Their results imply that the dynamics of gas in a halo are not significantly affected by the shock front unless the thermal energy of the ionized gas is greater than its gravitational potential energy. Furthermore, since gas in a halo is heated to the virial temperature even before reionization, the shock is weaker when the gas is ionized than a typical shock in the interstellar medium. Also, as noted above, the ionizing radiation reaching a given halo builds up in intensity over a considerable period of time. Thus we do not expect the ionization shock associated with the first encounter of ionizing radiation to have a large effect on the eventual fate of gas in the halo.

\section{RESULTS}

We assume the most popular cosmology to date (Garnavich et al. 1998), with $\Omega_{0}=0.3$ and $\Omega_{\Lambda}=0.7$. We illustrate the effects of cosmological parameters by displaying the results also for $\Omega_{0}=1$, and for $\Omega_{0}=0.3$ and $\Omega_{\Lambda}=0$. The models all assume $\Omega_{b} h^{2}=0.02$ and a Hubble constant $h=0.5$ if $\Omega_{0}=1$ and $h=0.7$ otherwise (where $H_{0}=100 h \mathrm{~km} \mathrm{~s}^{-1} \mathrm{Mpc}^{-1}$ ).

Figure 1 shows the temperature of the gas versus its baryonic overdensity $\Delta_{b}$ relative to the cosmic average (see Efstathiou 1992). The curves are for $z=8$ and assume $\Omega_{0}=0.3$ and $\Omega_{\Lambda}=0.7$. We include intergalactic radiation with a flux given by equation (6) for $\alpha=1.8$ and $n_{\gamma} / n_{b}=1$. The dotted curve shows $t_{H}=t_{\text {cool }}$ with no radiation field, where $t_{H}$ is the age of the universe, approximately equal to $6.5 \times 10^{9} h^{-1}(1+z)^{-3 / 2} \Omega_{0}^{-1 / 2} \mathrm{yr}$ at high redshift. This curve indicates the temperature to which gas has time to cool through atomic transitions before reionization. This temperature is always near $T=10^{4} \mathrm{~K}$ since below this temperature the gas becomes mostly neutral and the cooling time is very long. It is likely that only atomic cooling is relevant before reionization since molecular hydrogen is easily destroyed by even a weak ionizing background (Haiman et al. 1996a). The solid curve shows the equi-

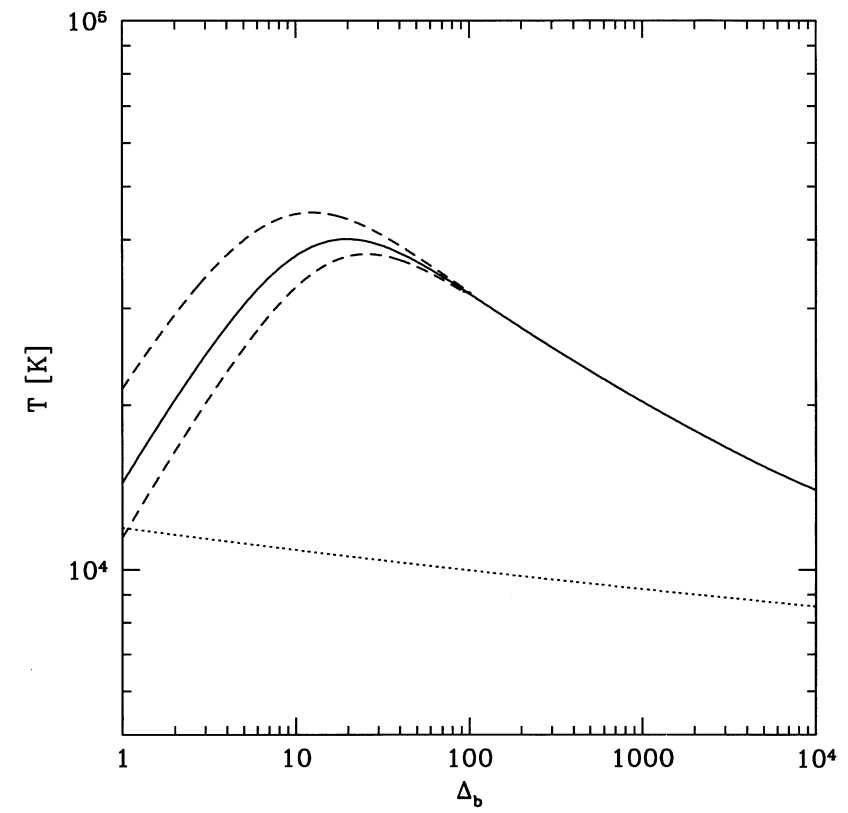

FIG. 1.-Temperature $T$ vs. baryon overdensity $\Delta_{b}: t_{H}=t_{\text {cool }}$, no radiation field (dotted curve); $t_{\text {heat }}=t_{\text {cool }}$, with radiation (solid curve); and $t_{H}$ equals the net cooling/heating time, with radiation (dashed curves). The curves assume $\Omega_{0}=0.3, \Omega_{\Lambda}=0.7, z=8, \alpha=1.8$, and $n_{\gamma} / n_{b}=1$. librium temperature for which the heating time $t_{\text {heat }}$ due to a UV radiation field equals the cooling time $t_{\text {cool }}$. The decrease in the temperature at $\Delta_{b}<10$ is due to the increased importance of Compton cooling, which is proportional to the gas density rather than its square. At a given density, gas is heated at reionization to the temperature indicated by the solid curve, unless the net cooling or heating time is too long. The dashed curves show the temperature where the net cooling or heating time equals $t_{H}$. By definition, points on the solid curve have an infinite net cooling or heating time, but there is also a substantial regime at low $\Delta_{b}$ where the net cooling or heating time is greater than $t_{H}$. However, this regime has only a minor effect on halos, since the mean overdensity inside the virial radius of a halo is of order 200 . On the other hand, if gas leaves the halo and expands, it quickly enters the regime where it cannot reach thermal equilibrium.

Figure 2 presents an example for the structure of a halo with an initial total mass of $M=3 \times 10^{7} M_{\odot}$ at $z=8$. We assume the same cosmological parameters as in Figure 1. The bottom plot shows the baryon overdensity $\Delta_{b}$ versus $r / r_{\text {vir }}$, and reflects our assumption of identical NFW profiles for both the dark matter and the baryons. The middle plot shows the neutral hydrogen fraction versus $r / r_{\text {vir }}$, and the top plot shows the ratio of thermal energy per particle $\left[\mathrm{TE}=(3 / 2) k_{B} T(r)\right]$ to potential energy per particle [PE $=\mu|\phi(r)|$, where $\phi(r)$ is the gravitational potential] versus $r / r_{\text {vir }}$. The dashed curves assume an optically thin halo, while the solid curves include radiative transfer and self-shielding. The self-shielded neutral core is apparent from the solid curves, but since the point where TE/PE $=1$ occurs outside this core, the overall unbound fraction does not depend strongly on the radiative transfer in this case. Its value is $67 \%$ assuming an optically thin halo and $64 \%$ when

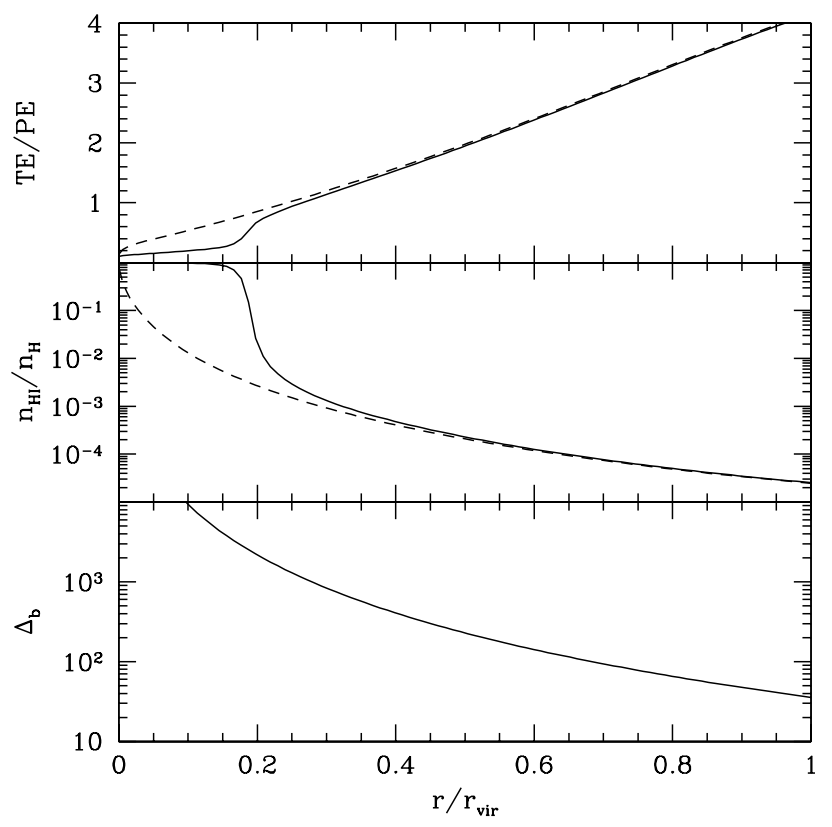

FIG. 2.- Halo structure after reionization, for a halo mass $M=3 \times 10^{7}$ $M_{\odot}$ at $z=8$ : baryon overdensity $\left(\Delta_{b}\right)$ vs. $r / r_{\mathrm{vir}}$ (bottom plot); neutral hydrogen fraction $\left(n_{\mathrm{H}} / n_{\mathrm{H}}\right)$ vs. $r / r_{\text {vir }}$ (middle plot); and the ratio of thermal energy (TE) to potential energy (PE) vs. $r / r_{\text {vir }}$ (top plot). The dashed curves assume an optically thin halo, while the solid curves include radiative transfer and self-shielding. All the curves assume $\Omega_{0}=0.3, \Omega_{\Lambda}=0.7$, $\alpha=1.8$, and $n_{\gamma} / n_{b}=1$. 
radiative transfer is included and only a fraction of the external photons make their way inside. Even when the opacity at the Lyman limit is large, some ionizing radiation still reaches the central parts of the halo because (1) the opacity drops quickly above the Lyman limit, and (2) the heated gas radiates ionizing photons inward.

Figure 3 shows the unbound gas fraction after reionization as a function of the total halo mass. We assume $\Omega_{0}=$ $0.3, \Omega_{\Lambda}=0.7$, and $n_{\gamma} / n_{b}=1$. The three pairs of curves shown consist of a solid line (which includes radiative transfer) and a dashed line (which assumes an optically thin halo). From right to left, the first pair is for $\alpha=1.8$ and $z=8$, the second is for $\alpha=5$ and $z=8$, and the third is for $\alpha=1.8$ and $z=20$. In each case the self-shielded core lowers the unbound fraction when we include radiative transfer (solid vs. dashed lines), particularly when the unbound fraction is sufficiently large that it includes part of the core itself. High-energy photons above the Lyman limit penetrate deep into the halo and heat the gas efficiently. Therefore, a steepening of the spectral slope from $\alpha=1.8$ to $\alpha=5$ decreases the temperature throughout the halo and lowers the unbound gas fraction. This is only partially compensated for by our UV flux normalization, which increases $I_{21}$ with increasing $\alpha$ so as to get the same density of ionizing photons in equation (7). Increasing the reionization redshift from $z=8$ to $z=20$ increases the binding energy of the gas, because the high-redshift halos are denser. Although the corresponding increase of $I_{21}$ with redshift (at a fixed $n_{\gamma} / n_{b}$ ) counteracts this change, the fraction of expelled gas is still reduced because of the deeper potential wells of higher redshift halos.

From plots similar to those shown in Figure 3, we find the total halo mass at which the unbound gas fraction is $50 \%$. We henceforth refer to this mass as the $50 \%$ mass. Figure 4 plots this mass as a function of the reionization redshift for different spectra and cosmological models. The

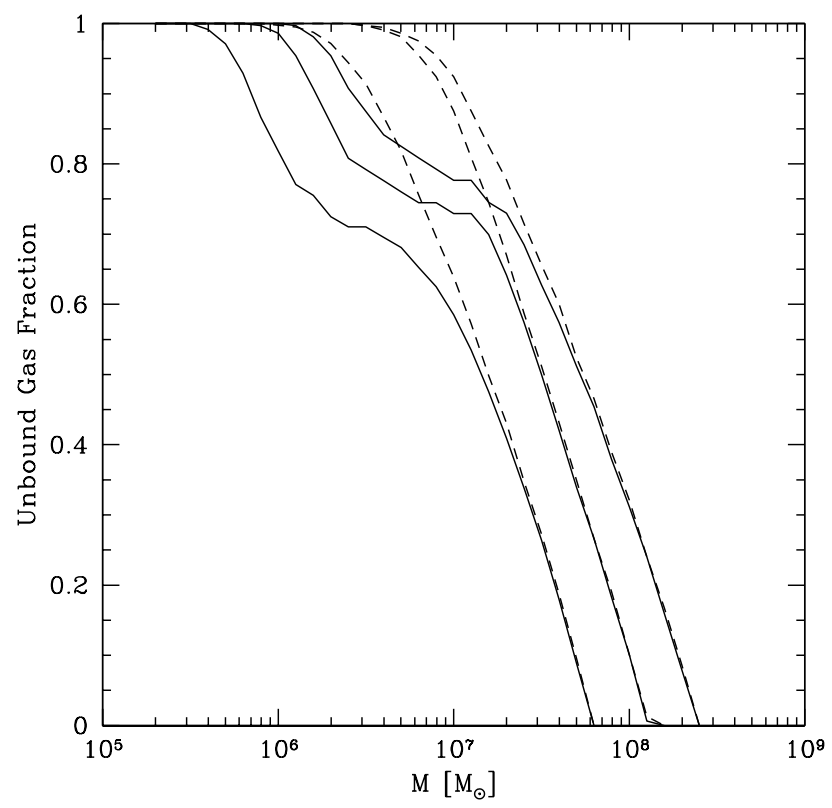

Fig. 3.-Unbound gas fraction vs. total halo mass. There are three pairs of curves, each consisting of a solid line (with radiative transfer) and a dashed line (without radiative transfer). From right to left, the three sets of curves correspond to $\alpha=1.8, z=8 ; \alpha=5, z=8$; and $\alpha=1.8, z=20$. All the curves assume $\Omega_{0}=0.3, \Omega_{\Lambda}=0.7$, and $n_{\gamma} / n_{b}=1$.

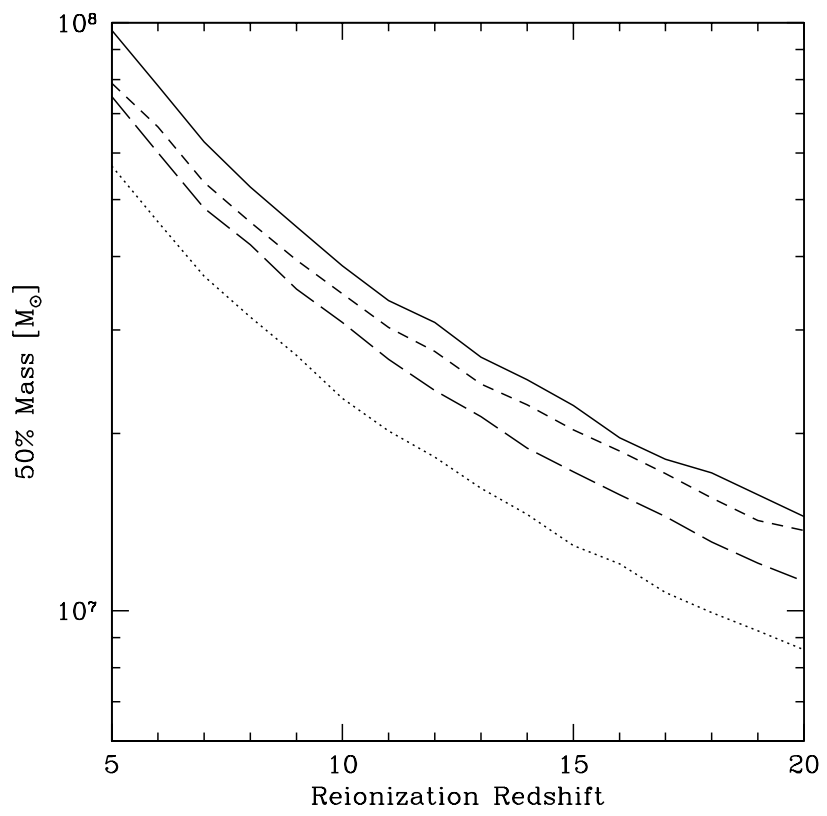

FIG. 4.-Total halo mass for which $50 \%$ of the gas is unbound vs. reionization redshift. The solid line assumes $\alpha=1.8$, and the dotted line assumes $\alpha=5$, both for an $\Omega_{0}=0.3, \Omega_{\Lambda}=0.7$ cosmology. The other lines assume $\alpha=1.8$ but different cosmologies. The short-dashed line assumes $\Omega_{0}=0.3, \Omega_{\Lambda}=0$ and the long-dashed line assumes $\Omega_{0}=1$. All assume $n_{\gamma} / n_{b}=1$.

solid line assumes $\alpha=1.8$ and the dotted line $\alpha=5$, both for $\Omega_{0}=0.3$ and $\Omega_{\Lambda}=0.7$. The other lines assume $\alpha=1.8$ but different cosmologies. The short-dashed line assumes $\Omega_{0}=0.3, \Omega_{\Lambda}=0$, and the long-dashed line assumes $\Omega_{0}=1$. All assume $n_{\gamma} / n_{b}=1$. Gas becomes unbound when its thermal energy equals its potential binding energy. The thermal energy depends on temperature, but the equilibrium temperature does not change much with redshift since we increase the UV flux normalization by the same $(1+z)^{3}$ factor as the mean baryonic density. With this prescription for the UV flux, the $50 \%$ mass occurs at a value of the circular velocity that is roughly constant with redshift. Thus, for each curve, the change in mass with redshift is mostly due to the change in the characteristic halo density, which affects the relation between circular velocity and mass.

The cosmological parameters have only a modest effect on the $50 \%$ mass and change it by up to $35 \%$ at a given redshift. Lowering $\Omega_{0}$ reduces the characteristic density of a halo of given mass, and so a higher mass is required in order to keep the gas bound. Adding a cosmological constant reduces the density further through $\Delta_{c}$ (see eqs. [A1] and [A2]). For the three curves with $\alpha=1.8$, the circular velocity of the $50 \%$ mass equals $13 \mathrm{~km} \mathrm{~s}^{-1}$ at all redshifts, up to variations of a few percent.

The spectral shape of the ionizing flux affects modestly the threshold circular velocity corresponding to the $50 \%$ mass, because assuming a steeper spectrum (i.e., with a larger $\alpha$ ) reduces the gas temperature and thus requires a shallower potential to keep the gas bound. A higher flux normalization has the opposite effect of increasing the threshold circular velocity. The left panel of Figure 5 shows the variation of circular velocity with spectral shape, for two normalizations $\left(n_{\gamma} / n_{b}=1\right.$ and $n_{\gamma} / n_{b}=0.1$, solid and dashed curves, respectively). The right panel shows the complemen- 


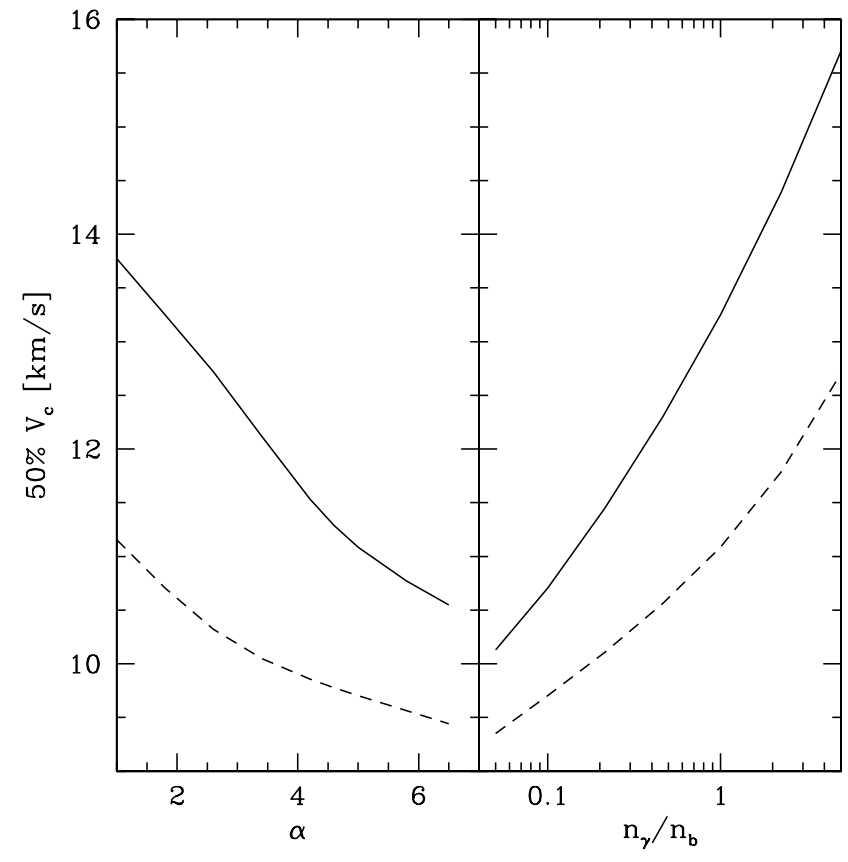

FIG. 5.-Circular halo velocity at which $50 \%$ of the gas is unbound, as a function of ionizing spectrum. Both panels show $V_{c}$ on the vertical axis. The left panel varies the spectral slope $\alpha$ for two values of the normalization, $n_{y} / n_{b}=1$ (solid curve) and $n_{\gamma} / n_{b}=0.1$ (dashed curve). The right panel varies the normalization for two spectral slopes, $\alpha=1.8$ (solid curve) and $\alpha=5$ (dashed curve). All curves assume an $\Omega_{0}=0.3, \Omega_{\Lambda}=0.7$ cosmology.

tary case of varying the spectral normalization, using two values for the spectral slope $(\alpha=1.8$ and $\alpha=5$, solid and dashed curves, respectively). All curves assume an $\Omega_{0}=0.3$, $\Omega_{\Lambda}=0.7$ cosmology.

Obviously, $50 \%$ is a fairly arbitrary choice for the unbound gas fraction at which halos evaporate. Figure 3 shows that for a given halo, the unbound gas fraction changes from $10 \%$ to $90 \%$ over a factor of $\sim 60$ in mass, or a factor of $\sim 4$ in velocity dispersion. When $50 \%$ of the gas is unbound, however, the rest of the gas is also substantially heated, and we expect the process of collapse and fragmentation to be inhibited. In the extreme case where the gas expands until a steady state is achieved where it is pressure confined by the IGM, less than $10 \%$ of the original gas is left inside the virial radius. However, continued infall of dark matter should limit the expansion. Numerical simulations may be used to define more precisely the point at which gas halos are disrupted. Clearly, photoevaporation affects even halos with masses well above the $50 \%$ mass, although these halos do not completely evaporate. Note that it is also clear from Figure 3 that not including radiative transfer would have only a minor effect on the value of the $50 \%$ mass (typically $\sim 5 \%$ ).

Given the values of the unbound gas fraction in halos of different masses, we can integrate to find the total gas fraction in the universe that becomes unbound at reionization. This calculation requires the abundance distribution of halos, which is readily provided by the Press-Schechter mass function for CDM cosmologies (relevant expressions are given, e.g., in NFW). The high-mass cutoff in the integration is given by the lowest mass halo for which the unbound gas fraction is zero, since halos above this mass are not significantly affected by the UV radiation. The low- mass cutoff is given by the lowest mass halo in which gas has assembled by the reionization redshift. We adopt for this low-mass cutoff the linear Jeans mass, which we calculate following Peebles (1993, that book's § 6). The gas temperature in the universe follows the cosmic microwave background temperature down to a redshift $1+z_{t} \sim$ $740\left(\Omega_{b} h^{2}\right)^{2 / 5}$, at which the baryonic Jeans mass is 1.9 $\times 10^{5}\left(\Omega_{b} h^{2}\right)^{-1 / 2} M_{\odot}$. After this redshift, the gas temperature goes down as $(1+z)^{2}$, so the baryon Jeans mass acquires a factor of $\left[(1+z) /\left(1+z_{t}\right)\right]^{3 / 2}$. Until now we have considered baryons only, but if we add dark matter, then the mean density (or the corresponding gravitational force) is increased by $\Omega_{0} / \Omega_{b}$, which decreases the baryonic Jeans mass by $\left(\Omega_{0} / \Omega_{b}\right)^{-3 / 2}$. The corresponding total halo mass is $\Omega_{0} / \Omega_{b}$ times the baryonic mass. Thus the Jeans cutoff before reionization corresponds to a total halo mass of

$$
M_{\mathrm{J}}=6.9 \times 10^{3}\left(\frac{\Omega_{0} h^{2}}{0.2}\right)^{-1 / 2}\left(\frac{\Omega_{b} h^{2}}{0.02}\right)^{-3 / 5}\left(\frac{1+z}{10}\right)^{3 / 2} M_{\odot} .
$$

This value agrees with the numerical spherical collapse calculations of Haiman et al. (1996b).

We thus calculate the total fraction of gas in the universe that is bound in preexisting halos, and the fraction of this gas which then becomes unbound at reionization. In Figure 6 we show the fraction of the collapsed gas which evaporates as a function of the reionization redshift. The solid line assumes $\alpha=1.8$, and the dotted line assumes $\alpha=5$, both for $\Omega_{0}=0.3, \Omega_{\Lambda}=0.7$. The other lines assume $\alpha=1.8$, the short-dashed line with $\Omega_{0}=0.3, \Omega_{\Lambda}=0$ and the longdashed line with $\Omega_{0}=1$. All assume $n_{\gamma} / n_{b}=1$ and a primordial $n=1$ (scale-invariant) power spectrum. In each case we normalized the CDM power spectrum to the present cluster abundance, $\sigma_{8}=0.5 \Omega_{0}^{-0.5}$ (see, e.g., Pen 1998), where $\sigma_{8}$ is

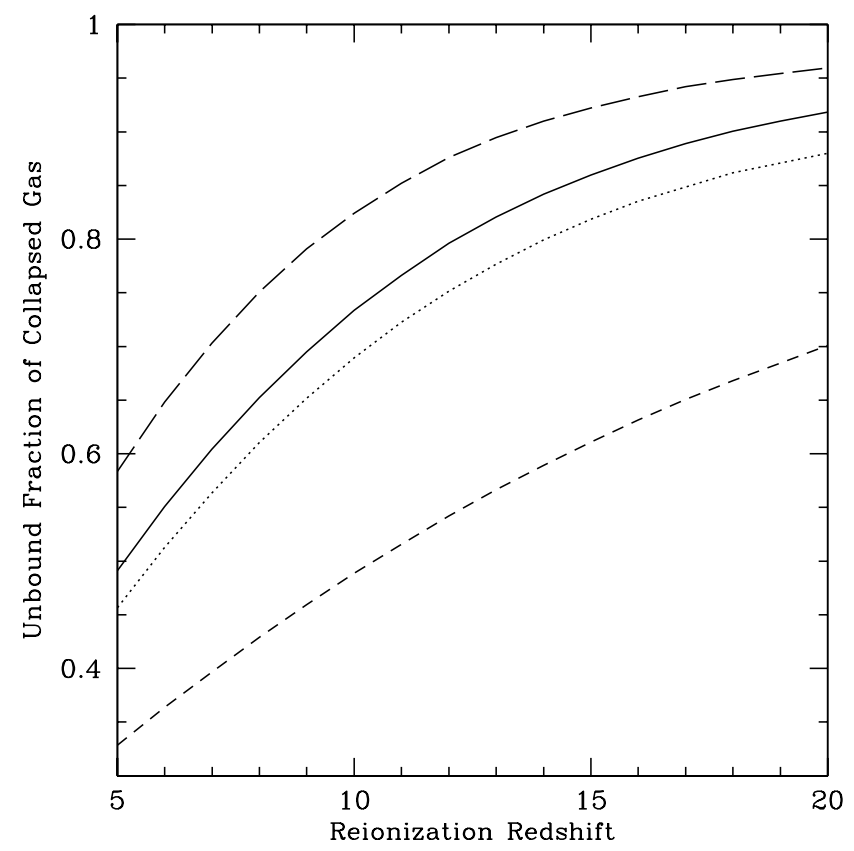

Fig. 6.-Fraction of the collapsed gas that evaporates from halos at reionization vs. the reionization redshift. The solid line assumes $\alpha=1.8$, and the dotted line assumes $\alpha=5$, both for an $\Omega_{0}=0.3, \Omega_{\Lambda}=0.7$ cosmology. The other lines assume $\alpha=1.8$ but different cosmologies. The shortdashed line assumes $\Omega_{0}=0.3, \Omega_{\Lambda}=0$, and the long-dashed line assumes $\Omega_{0}=1$. All assume $n_{\gamma} / n_{b}=1$. 
the rms amplitude of mass fluctuations in spheres of radius $8 h^{-1} \mathrm{Mpc}$. The fraction of collapsed gas that is unbound is $\sim 0.4-0.7$ at $z=6$, and it increases with redshift. This fraction clearly depends strongly on the halo abundance but is relatively insensitive to the spectral slope $\alpha$ of the ionizing radiation. In hierarchical models, the characteristic mass (and binding energy) of virialized halos is smaller at higher redshifts, and a larger fraction of the collapsed gas therefore escapes once it is photoheated. Among the three cosmological models, the characteristic mass at a given redshift is smallest for $\Omega_{0}=1$ and largest for $\Omega_{0}=0.3, \Omega_{\Lambda}=0$.

In Figure 7 we show the total fraction of gas in the universe that evaporates at reionization. The solid line assumes $\alpha=1.8$, and the dotted line assumes $\alpha=5$, both for $\Omega_{0}=$ $0.3, \Omega_{\Lambda}=0.7$. The other lines assume $\alpha=1.8$, the shortdashed line with $\Omega_{0}=0.3, \Omega_{\Lambda}=0$ and the long-dashed line with $\Omega_{0}=1$. All assume $n_{\gamma} / n_{b}=1$. For the different cosmologies, the total unbound fraction goes up to $20 \%-25 \%$ if reionization occurs as late as $z=6-7$; in this case a substantial fraction of the total gas in the universe undergoes the process of expulsion from halos. However, this fraction typically decreases at higher redshifts. Although a higher fraction of the collapsed gas evaporates at higher $z$ (see Fig. 6), a smaller fraction of the gas in the universe lies in halos in the first place. The latter effect dominates except for the open model up to $z \sim 7$. As is well known, the $\Omega_{0}=1$ model produces late structure formation, and indeed the collapsed fraction decreases rapidly with redshift in this cosmological model. The low- $\Omega_{0}$ models approach the $\Omega_{0}=1$ behavior at high $z$, but this occurs faster for the flat model with a cosmological constant than for the open model with the same value of $\Omega_{0}$.

Changing the dark matter and gas profiles as discussed in $\S 2$ has a modest effect on the results. For example, with $\Omega_{0}=0.3, \Omega_{\Lambda}=0.7$, and $z=8$, and for our standard model where the gas and dark matter follow identical NFW pro-

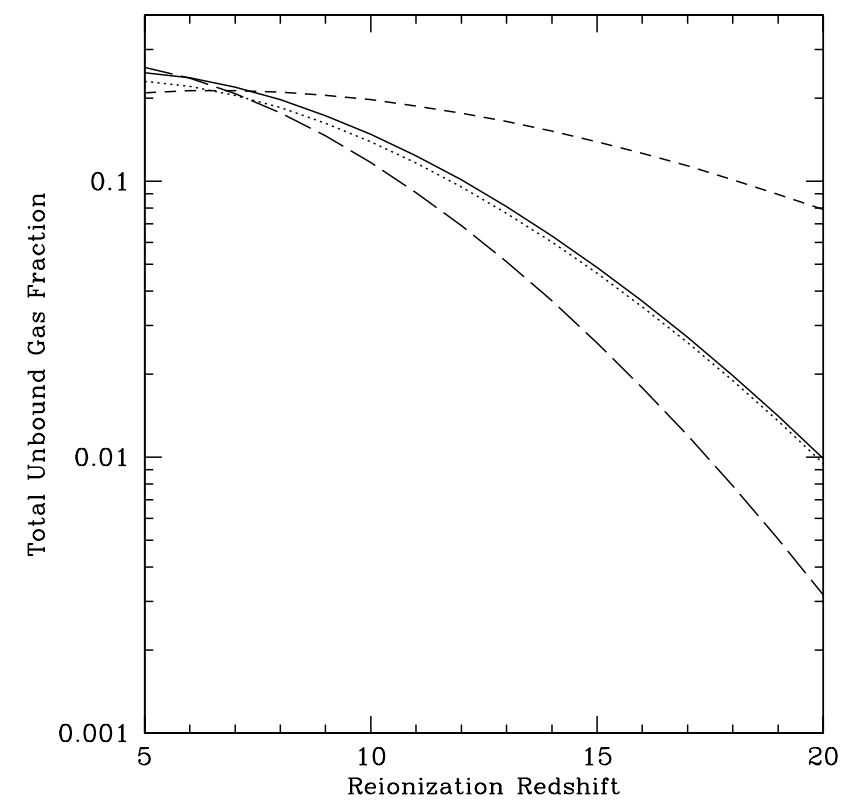

FIG. 7.- Total fraction of gas in the universe which evaporates from halos at reionization, vs. the reionization redshift. The solid line assumes $\alpha=1.8$, and the dotted line assumes $\alpha=5$, both for an $\Omega_{0}=0.3, \Omega_{\Lambda}=0.7$ cosmology. The other lines assume $\alpha=1.8$ but different cosmologies. The short-dashed line assumes $\Omega_{0}=0.3, \Omega_{\Lambda}=0$ and the long-dashed line assumes $\Omega_{0}=1$. All assume $n_{\gamma} / n_{b}=1$. files, the total unbound gas fraction is $19.8 \%$, and the halo mass that loses $50 \%$ of its baryons is $5.25 \times 10^{7} M_{\odot}$. If we let the mass and the baryons follow the profile of equation (4), the corresponding results are $20.0 \%$ and $5.31 \times 10^{7} M_{\odot}$ for $b=10$ in equation (4) and $20.9 \%$ and $6.84 \times 10^{7} M_{\odot}$ for $b=5$ (i.e., a larger core). With an NFW mass profile but gas in hydrostatic equilibrium at the virial temperature, the unbound fraction is $19.2 \%$, and the $50 \%$ mass is 4.33 $\times 10^{7} M_{\odot}$. If we let the gas temperature be $T=2 T_{\text {vir }}$, the unbound fraction is $22.0 \%$, and the $50 \%$ mass is 1.18 $\times 10^{8} M_{\odot}$. For clouds of gas that condense by cooling for a Hubble time, the unbound fraction is $18.2 \%$, and the $50 \%$ mass is $3.38 \times 10^{7} M_{\odot}$. We conclude that centrally concentrated gas clouds are in general more effective at retaining their gas, but the effect on the overall unbound gas fraction in the universe is modest, even for large variations in the profile. If we return to the NFW profile but adopt $f=0.01$ instead of $f=0.5$ in the NFW prescription for finding the collapse redshift (see Appendix A), we find an unbound fraction of $20.3 \%$ and a $50 \%$ mass of $6.06 \times 10^{7} M_{\odot}$. Finally, lowering $\Omega_{b}$ by a factor of 2 changes the unbound fraction to $19.0 \%$ and the $50 \%$ mass to $5.44 \times 10^{7} M_{\odot}$. Our predictions appear to be robust against variations in the model parameters.

\section{IMPLICATIONS FOR THE IGM AND FOR LOW-REDSHIFT OBJECTS}

Our calculations show that a substantial fraction of gas in the universe may lie in virialized halos before reionization and that most of it evaporates out of the halos when it is photoionized and heated at reionization. The resulting outflows of gas from halos may have interesting implications for the subsequent evolution of structure in the IGM. We discuss some of these implications in this section.

In the pre-reionization epoch, a fraction of the gas in the dense cores of halos may fragment and form stars. Some star formation is, of course, needed in order to produce the ionizing flux that leads to reionization. These Population III stars produce the first metals in the universe, and they may make a substantial contribution to the enrichment of the IGM. Numerical models by Mac Low \& Ferrara (1999) suggest that feedback from supernovae is very efficient at expelling metals from dwarf galaxies of total mass $3.5 \times 10^{8}$ $M_{\odot}$, although it ejects only a small fraction of the interstellar medium in these hosts. Obviously, the metal expulsion efficiency depends on the presence of clumps in the supernova ejecta (Franco et al. 1993) and on the supernova rate, the latter depending on the unknown star formation rate and the initial mass function of stars at high redshifts. Reionization provides an alternative method for expelling metals efficiently out of dwarf galaxies by directly photoheating the gas in their halos, leading to its evaporation along with its metal content. ${ }^{1}$

Gas that falls into halos and is expelled at reionization attains a different entropy than if it had stayed at the mean density of the universe. Gas that collapses into a halo is at a high overdensity when it is photoheated, and it is therefore at a lower entropy than if it were heated to the same tem-

\footnotetext{
${ }^{1}$ Note that we have assumed zero metallicity in calculating cooling. Even if some metals had already been mixed into the IGM, the metallicity of newly formed objects was likely too low to affect cooling since even at $z \sim 3$ the typical metallicity of the Ly $\alpha$ forest has been observed to be $<0.01$ solar (Songaila \& Cowie 1996; Tytler et al. 1995).
} 
perature at the mean cosmic density. However, the overall change in the entropy density of the IGM is small for two reasons. First, even at $z=6$ only about $25 \%$ of the gas in the universe undergoes evaporation. Second, the gas remains in ionization equilibrium and is photoheated during its initial expansion. For example, if $z=6, \Omega_{0}=0.3$, $\Omega_{\Lambda}=0.7, n_{\gamma} / n_{b}=1$, and $\alpha=1.8$, then the recombination time becomes longer than the dynamical time only when the gas expands down to an overdensity of 26, at which point its temperature is $22,400 \mathrm{~K}$ compared with an initial (nonequilibrium) temperature of $19,900 \mathrm{~K}$ for gas at the mean density. The resulting overall reduction in the entropy is the same as would be produced by reducing the temperature of the entire IGM by a factor of 1.6. This factor reduces to 1.4 if we increase $z$ to 8 or increase $\alpha$ to 5 . Note that Haehnelt \& Steinmetz (1998) showed that differences in temperature by a factor of 3-4 result in possibly observable differences in the Doppler parameter distribution of $\mathrm{Ly} \alpha$ absorption lines at redshifts 3-5.

When the halos evaporate, recombinations in the gas could produce $\mathrm{Ly} \alpha$ lines or radiation from two-photon transitions to the ground state of hydrogen. However, a simple estimate shows that the resulting luminosity is too small for direct detection unless these halos are illuminated by an internal ionizing source. In an externally illuminated, $z=6,10^{8} M_{\odot}$ halo, our calculations imply a total of $\sim 1 \times 10^{50}$ recombinations per second. Note that the number of recombinations is dominated by the high-density core, and if we did not include self-shielding we would obtain an overestimate by a factor of $\sim 15$. If each recombination releases one or two photons with a total energy of $10.2 \mathrm{eV}$, then for $\Omega_{0}=0.3$ and $\Omega_{\Lambda}=0.7$ the observed flux is $\sim 5 \times 10^{-20}$ ergs $\mathrm{s}^{-1} \mathrm{~cm}^{-2}$. This flux is well below the sensitivity of the planned Next Generation Space Telescope, even if part of this flux is concentrated in a narrow line.

The photoionization heating of the gaseous halos of dwarf galaxies resulted in outflows with a characteristic velocity of $\sim 20-30 \mathrm{~km} \mathrm{~s}^{-1}$. These outflows must have induced peculiar velocities of a comparable magnitude in the IGM surrounding these galaxies. The effect of the outflows on the velocity field and entropy of the IGM at $z=5-10$ could in principle be searched for in the absorption spectra of high-redshift sources, such as quasars. These small-scale fluctuations in velocity and the resulting temperature fluctuations have been seen in recent simulations by Bryan et al. (1998). However, the small halos responsible for these outflows were only barely resolved even in these high-resolution simulations of a small volume.

The evaporating galaxies could contribute to the high column density end of the Ly $\alpha$ forest (see Bond et al. 1988). For example, shortly after being photoionized, a $z=8,5$ $\times 10^{7} M_{\odot}$ halo has a neutral hydrogen column density of $2 \times 10^{16} \mathrm{~cm}^{-2}$ at an impact parameter of $0.5 r_{\mathrm{vir}}=0.66 \mathrm{kpc}$, $6 \times 10^{17} \mathrm{~cm}^{-2}$ at $0.25 r_{\text {vir }}$, and $9 \times 10^{20} \mathrm{~cm}^{-2}$ (or $9 \times 10^{18}$ $\mathrm{cm}^{-2}$ if we do not include self-shielding) at $0.1 r_{\text {vir }}$ (assuming $\Omega_{0}=0.3, \Omega_{\Lambda}=0.7, \alpha=1.8$, and $n_{\gamma} / n_{b}=1$ ). These column densities will decline as the gas expands out of the host galaxy. Abel \& Mo (1998) have suggested that a large fraction of the Lyman limit systems at $z \sim 3$ may correspond to minihalos that survived reionization. Remnant absorbers due to galactic outflows can be distinguished from largescale absorbers in the IGM by their compactness. Close lines of sight due to quasar pairs or gravitational lensed quasars (see, e.g., Crotts \& Fang 1998; Petry, Impey, \&
Foltz 1998, and references therein) should probe different $\mathrm{H}$ I column densities in galactic outflow absorbers but similar column densities in the larger, more common absorbers. Follow-up observations with high spectroscopic resolution could reveal the velocity fields of these outflows.

Although much of the gas in the universe evaporated at reionization, the underlying dark matter halos continued to evolve through infall and merging, and the heated gas may have accumulated in these halos at lower redshifts. This latter process has been discussed by a number of authors, with an emphasis on the effect of reionization and the resulting heating of gas. Thoul \& Weinberg (1996) found a reduction of $\sim 50 \%$ in the collapsed gas mass due to heating, for a halo of $V_{c}=50 \mathrm{~km} \mathrm{~s}^{-1}$ at $z=2$, and a complete suppression of infall below $V_{c}=30 \mathrm{~km} \mathrm{~s}^{-1}$. The effect is thus substantial on halos with virial temperatures well above the gas temperature. Their interpretation is that pressure support delays turnaround substantially and slows the subsequent collapse. Indeed, as noted in $\S 2$, the ratio of the pressure force to the gravitational force on the gas is roughly equal to the ratio of its thermal energy to its potential energy. For a given enclosed mass, the potential energy of a shell of gas increases as its radius decreases. Before collapse, each gas shell expands with the Hubble flow until its expansion is halted and then reversed. Near turnaround, the gas is weakly bound, and the pressure gradient may prevent collapse even for gas below the halo virial temperature. On the other hand, gas that is already well within the virial radius is tightly bound, which explains our lower value of $V_{c} \sim 13 \mathrm{~km} \mathrm{~s}^{-1}$ for halos that lose half their gas at reionization.

Three-dimensional numerical simulations (Quinn et al. 1996; Weinberg et al. 1997; Navarro \& Steinmetz 1997) have also explored the question of whether dwarf galaxies could re-form at $z \gtrsim 2$. The heating by the UV background was found to suppress infall of gas into even larger halos $\left(V_{c} \sim 75 \mathrm{~km} \mathrm{~s}^{-1}\right)$, depending on the redshift and on the ionizing radiation intensity. Navarro \& Steinmetz (1997) noted that photoionization reduces the cooling efficiency of gas at low densities, which suppresses further the late infall at redshifts below 2 . We note that these various simulations assume an isotropic ionizing radiation field and do not calculate radiative transfer. Photoevaporation of a gas cloud has been calculated in a two-dimensional simulation (Shapiro, Raga, \& Mellema 1998), and methods are being developed for incorporating radiative transfer into threedimensional cosmological simulations (e.g., Abel, Norman, \& Madau 1999; Razoumov \& Scott 1999).

Our results have interesting implications for the fate of gas in low-mass halos. Gas evaporates at reionization from halos below $V_{c} \sim 13 \mathrm{~km} \mathrm{~s}^{-1}$, or a velocity dispersion $\sigma \sim 10$ $\mathrm{km} \mathrm{s}^{-1}$. A similar value of the velocity dispersion is also required to reach a virial temperature of $10^{4} \mathrm{~K}$, allowing atomic cooling and perhaps star formation before reionization. Thus halos with $\sigma \gtrsim 10 \mathrm{~km} \mathrm{~s}^{-1}$ could have formed stars before reionization. They would have kept their gas after reionization and could have had ongoing star formation subsequently. These halos were the likely sites of Population III stars and could have been the progenitors of dwarf galaxies in the local universe (see Miralda-Escude \& Rees 1998). On the other hand, halos with $\sigma \lesssim 10 \mathrm{~km} \mathrm{~s}^{-1}$ could not have cooled before reionization. Their warm gas was completely evaporated from them at reionization and could not have returned to them until very low redshifts, 
possibly $z \lesssim 1$, so that their stellar population should be relatively young.

It is interesting to compare these predictions with the properties of dwarf spheroidal galaxies in the Local Group that have low central velocity dispersions. At first sight this appears to be a difficult task. The dwarf galaxies vary greatly in their properties, with many showing evidence for multiple episodes of star formation as well as some very old stars (see the recent review by Mateo 1998). Another obstacle is the low temporal resolution of age indicators for old stellar populations. For example, if $\Omega_{0}=0.3$ and $\Omega_{\Lambda}=0.7$, then the age of the universe is $43 \%$ of its present age at $z=1$ and $31 \%$ at $z=1.5$. Thus stars that formed at these redshifts may already be $\sim 10$ Gyr old at present, and are difficult to distinguish from stars that formed at $z>5$.

Nevertheless, one of our robust predictions is that most early halos with $\sigma \lesssim 10 \mathrm{~km} \mathrm{~s}^{-1}$ could not have formed stars in the standard hierarchical scenario. Globular clusters belong to one class of objects with such a low-velocity dispersion. Peebles \& Dicke (1968) originally suggested that globular clusters may have formed at high redshifts, before their parent galaxies. However, in current cosmological models, most mass fluctuations on globular cluster scales were unable to cool effectively and fragment until $z \sim 10$ and were evaporated subsequently by reionization. We note that Fall \& Rees (1985) proposed an alternative formation scenario for globular clusters involving a thermal instability inside galaxies with properties similar to those of the Milky Way. Globular clusters have also been observed to form in galaxy mergers (e.g., Miller et al. 1997). It is still possible that some of the very oldest and most metal-poor globular clusters originated from $z \gtrsim 10$, before the UV background had become strong enough to destroy the molecular hydrogen in them. However, primeval globular clusters should have retained their dark halos, but observations suggest that globular clusters are not embedded in dark matter halos (Moore 1996; Heggie \& Hut 1995).

Another related population is the nine dwarf spheroidals in the Local Group with central velocity dispersions $\sigma \lesssim 10$ $\mathrm{km} \mathrm{s}^{-1}$, including five below $7 \mathrm{~km} \mathrm{~s}^{-1}$ (e.g., Mateo 1998). In the hierarchical clustering scenario, the dark matter in a present halo was most probably divided at reionization among several progenitors, which have since merged. The velocity dispersions of these progenitors were likely even lower than that of the final halo. Thus the dwarf galaxies could not have formed stars at high redshifts, and their formation presents an intriguing puzzle. There are two possible solutions to this puzzle, (1) the ionizing background dropped dramatically at low redshifts, allowing the dwarf galaxies to form at $z \lesssim 1$, or (2) the measured stellar velocity dispersions of the dwarf galaxies are well below the velocity dispersions of their dark matter halos.

Unlike globular clusters, the dwarf spheroidal galaxies are dark matter-dominated. The dark halo of a present-day dwarf galaxy may have virialized at high redshifts but accreted its gas at low redshift from the IGM. However, for dark matter halos accumulating primordial gas, Kepner et al. (1997) found that even if $I_{21}(z)$ declines as $(1+z)^{4}$ below $z=3$, only halos with $V_{c} \gtrsim 20 \mathrm{~km} \mathrm{~s}^{-1}$ can form atomic hydrogen by $z=1$, and $V_{c} \gtrsim 25 \mathrm{~km} \mathrm{~s}^{-1}$ is required to form molecular hydrogen.

Alternatively, the dwarf dark halos could have accreted cold gas at low redshift from a larger host galaxy rather than from the IGM. As long as the dwarf halos join their host galaxy at a redshift much lower than their formation redshift, they will survive disruption because of their high densities. The subsequent accretion of gas could result from passages of the dwarf halos through the gaseous tidal tail of a merger event or through the disk of the parent galaxy. In this case, retention of cold, dense, and possibly metalenriched gas against heating by the UV background requires a shallower potential well than accumulating warm gas from the IGM. Simulations of galaxy encounters (Barnes \& Hernquist 1992; Elmegreen, Kaufman, \& Thomasson 1993) have found that dwarf galaxies could form but with small amounts of dark matter. However, the initial conditions of these simulations assumed parent galaxies with a smooth dark matter distribution rather than clumpy halos with dense subhalos inside them. Simulations by Klypin et al. (1999) suggest that galaxy halos may have large numbers of dark matter satellites, most of which have no associated stars. If true, this implies that the dwarf spheroidal galaxies might be explained even if only a small fraction of dwarf dark halos accreted gas and formed stars.

A common origin for the Milky Way's dwarf satellites (and a number of halo globular clusters), as remnants of larger galaxies accreted by the Milky Way galaxy, has been suggested on independent grounds. These satellites appear to lie along two (e.g., Majewski 1994) or more (Lynden-Bell \& Lynden-Bell 1995; Fusi-Pecci et al. 1995) polar great circles. The star formation history of the dwarf galaxies (e.g., Grebel 1998) constrains their merger history and implies that the fragmentation responsible for their appearance must have occurred early in order to be consistent with the variation in stellar populations among the supposed fragments (Unavane, Wyse, \& Gilmore 1996; Olszewski 1998). Observations of interacting galaxies (outside the Local Group) also suggest the formation of "tidal dwarf galaxies" (e.g., Duc \& Mirabel 1997).

Finally, there exists the possibility that the measured velocity dispersion of stars in the dwarf spheroidals underestimates the velocity dispersion of their dark halos. Assuming that the stars are in equilibrium, their velocity dispersion could be lower than that of the halo if the mass profile is shallower than isothermal beyond the stellar core radius. As discussed in $\S 2$, halo profiles are thought to vary from being shallow in a central core to being steeper than isothermal at larger distances. The velocity dispersion and mass to light ratio of a dwarf spheroidal could also appear high if it is nonspherical or the stellar orbits are anisotropic. Some dwarf spheroidals may even not be dark matterdominated if they are tidally disrupted (e.g., Kroupa 1997). The observed properties of dwarf spheroidals require a central mass density of order $0.1 M_{\odot} \mathrm{pc}^{-3}$ (e.g., Mateo 1998), which is $\sim 7 \times 10^{5}$ times the present critical density. The stars therefore reside either in high-redshift halos or in the very central parts of low-redshift halos. Detailed observations of the velocity dispersion profiles of these stars could be used to discriminate between these possibilities.

\section{CONCLUSIONS}

We have shown that the photoionizing background radiation that filled the universe during reionization likely boiled most of the virialized gas out of CDM halos at that time. The evaporation process probably lasted of order a Hubble time because of the gradual increase in the UV background as the $\mathrm{H}$ II regions around individual sources overlapped and percolated until the radiation field inside 
them grew up to its cosmic value - amounting to the full contribution of sources from the entire Hubble volume. The precise reionization history depends on the unknown star formation efficiency and the potential existence of miniquasars in newly formed halos (Haiman \& Loeb 1998).

The total fraction of the cosmic baryons which participate in the evaporation process depends on the reionization redshift, the ionizing intensity, and the cosmological parameters, but it is not very sensitive to the precise gas and dark matter profiles of the halos. The central core of halos is typically shielded from the external ionizing radiation by the surrounding gas, but this core typically contains $<20 \%$ of the halo gas and has only a weak effect on the global behavior of the gas. We have found that halos are disrupted up to a circular velocity $V_{c} \sim 13 \mathrm{~km} \mathrm{~s}^{-1}$ for a shallow, quasar-like spectrum, or $V_{c} \sim 11 \mathrm{~km} \mathrm{~s}^{-1}$ for a stellar spectrum, assuming the photoionizing sources build up a density of ionizing photons comparable to the mean cosmological density of baryons. At this photoionizing intensity, the value of the circular velocity threshold is nearly independent of redshift. The corresponding halo mass changes, however, from $\sim 10^{8} M_{\odot}$ at $z=5$ to $\sim 10^{7} M_{\odot}$ at $z=20$, assuming a shallow ionizing spectrum.

Based on these findings, we expect that both globular clusters and Local Group dwarf galaxies with velocity dispersions $\lesssim 10 \mathrm{~km} \mathrm{~s}^{-1}$ formed at low redshift, most probably inside larger galaxies. The latter possibility has been suggested previously for the Milky Way's dwarf satellites based on their location along polar great circles.

We are grateful to Jordi Miralda-Escudé, Chris McKee, Roger Blandford, Lars Hernquist, David Spergel, and Jim Peebles for useful discussions. We also thank Renyue Cen and Jordi Miralda-Escude for assistance with the reaction and cooling rates. R. B. acknowledges support from Institute Funds. This work was supported in part by the NASA NAG 5-7039 grant (for A. L.).

\section{APPENDIX A}

\section{HALO PROFILE}

We follow the prescription of NFW for obtaining the density profiles of dark matter halos, but instead of adopting a constant overdensity of 200 , we use the fitting formula of Bryan \& Norman (1998) for the virial overdensity,

$$
\Delta_{c}=18 \pi^{2}+82 d-39 d^{2}
$$

for a flat universe with a cosmological constant and

$$
\Delta_{c}=18 \pi^{2}+60 d-32 d^{2}
$$

for an open universe, where $d \equiv \Omega(z)-1$. Given $\Omega_{0}$ and $\Omega_{\Lambda}$, we define

$$
\Omega(z)=\frac{\Omega_{0}(1+z)^{3}}{\Omega_{0}(1+z)^{3}+\Omega_{\Lambda}+\left(1-\Omega_{0}-\Omega_{\Lambda}\right)(1+z)^{2}} .
$$

In equation (3), $c$ is determined for a given $\delta_{c}$ by the relation

$$
\delta_{c}=\frac{\Delta_{c}}{3} \frac{c^{3}}{\ln (1+c)-c /(1+c)} .
$$

The characteristic density is given by

$$
\delta_{c}=C(f) \Omega(z)\left(\frac{1+z_{\mathrm{coll}}}{1+z}\right)^{3} .
$$

For a given halo of mass $M$, the collapse redshift $z_{\text {coll }}$ is defined as the time at which a mass $M / 2$ was first contained in progenitors more massive than some fraction $f$ of $M$. This is computed using the extended Press-Schechter formalism (e.g., Lacey \& Cole 1993). NFW find that $f=0.01$ fits their $z=0$ simulation results best. Since we are interested in high redshifts when mergers are very frequent, we adopt the more natural $f=0.5$ but also check the $f=0.01$ case. (For example, the survival time of a $z=8,5 \times 10^{7} M_{\odot}$ halo before it merges is $\sim 30 \%-40 \%$ of the age of the universe at that redshift [Lacey \& Cole 1993].) In both cases we adopt the normalization of NFW, which is $C(0.5)=2 \times 10^{4}$ and $C(0.01)=3 \times 10^{3}$.

\section{APPENDIX B}

\section{RADIATIVE TRANSFER}

We neglect atomic transitions of helium atoms in the radiative transfer calculation. We only consider halos for which $k_{B} T$ is well below the ionization energy of hydrogen, and so following Tajiri \& Umemura (1998) we assume that recombinations to excited levels do not result in further ionizations. On the other hand, recombinations to the ground state result in the emission of ionizing photons all of which are in a narrow frequency band just above the Lyman limit frequency $v=v_{\mathrm{L}}$. We follow separately these emitted photons and the external incoming radiation. The external photons undergo absorption with an 
optical depth at the Lyman limit determined by

$$
\frac{d \tau_{v_{\mathrm{L}}}}{d s}=\sigma_{\mathrm{HI}}\left(v_{\mathrm{L}}\right) n_{\mathrm{H} \mathrm{I}} .
$$

The emitted photons near $v_{\mathrm{L}}$ are propagated by the equation of radiative transfer,

$$
\frac{d I_{v}}{d s}=-\sigma_{\mathrm{HI}}(v) n_{\mathrm{HI}} I_{v}+\eta_{v} .
$$

Assuming all emitted photons are just above $v=v_{\mathrm{L}}$, we can set $\sigma_{\mathrm{H}}(v)=\sigma_{\mathrm{H}}\left(v_{\mathrm{L}}\right)$ in this equation and propagate the total number flux of ionizing photons,

$$
F_{1} \equiv \int_{v_{\mathrm{L}}}^{\infty} \frac{I_{v}}{h v} d v
$$

The emissivity term for this quantity is

$$
\int_{v_{\mathrm{L}}}^{\infty} \frac{\eta_{v}}{h v} d v=\frac{\omega}{4 \pi} \alpha_{\mathrm{HI}} n_{\mathrm{H} \text { II }} n_{e}
$$

where $\alpha_{\mathrm{H}}$ is the total recombination coefficient to all bound levels of hydrogen and $\omega$ is the fraction of recombinations to the ground state. In terms of Table 5.2 of Spitzer (1978), $\omega=\left(\phi_{1}-\phi_{2}\right) / \phi_{1}$. We find that a convenient fitting formula up to 64,000 $\mathrm{K}$, accurate to $2 \%$, is (with $T$ in $\mathrm{K}$ )

$$
\omega=0.205-0.0266 \ln (T)+0.0049 \ln ^{2}(T) .
$$

When these photons are emitted, they carry away the kinetic energy of the absorbed electron. When the photons are reabsorbed at some distance from where they were emitted, they heat the gas with this extra energy. Since $k_{B} T \ll h v_{\mathrm{L}}$, we do not need to compute the exact frequency distribution of these photons. Instead we solve a single radiative-transfer equation for the total flux of energy (above the ionization energy of hydrogen) in these photons,

The emissivity term for radiative transfer of $F_{2}$ is

$$
F_{2} \equiv \int_{v_{\mathrm{L}}}^{\infty} \frac{I_{v}}{h v}\left(h v-h v_{\mathrm{L}}\right) d v .
$$

$$
\int_{v_{\mathrm{L}}}^{\infty} \frac{\eta_{v}}{h v}\left(h v-h v_{\mathrm{L}}\right) d v=\frac{2.07 \times 10^{-11}}{T^{1 / 2}} \frac{\chi_{1}(\beta)-\chi_{2}(\beta)}{4 \pi} n_{\mathrm{H}} n_{e} \operatorname{ergs~cm}^{-3} \mathrm{~s}^{-1} \mathrm{sr}^{-1}
$$

where $\beta=h v_{\mathrm{L}} / k T, T$ is in $\mathrm{K}$ and the functions $\chi_{1}$ and $\chi_{2}$ are given in Table 6.2 of Spitzer (1978). We find a fitting formula up to $64,000 \mathrm{~K}$, accurate to $2 \%$ (with $T$ in $\mathrm{K}$ ):

$$
\chi_{1}(T)-\chi_{2}(T)= \begin{cases}0.78, & \text { if } T<10^{3} \quad \mathrm{~K} \\ -0.172+0.255 \ln (T)-0.0171 \ln ^{2}(T), & \text { otherwise }\end{cases}
$$

From each point we integrate along all lines of sight to find $\tau_{v_{\mathrm{L}}}, F_{1}$, and $F_{2}$ as a function of angle. Because of spherical symmetry, we do this only at each radius, and the angular dependence only involves $\theta$, the angle relative to the radial direction. We then integrate to find the photoionization rate. For each atomic species, the rate is

$$
\Gamma_{\gamma i}=\int_{0}^{4 \pi} d \Omega \int_{v_{i}}^{\infty} \frac{I_{v}}{h v} \sigma_{i}(v) d v \mathrm{~s}^{-1}
$$

where $v_{i}$ and $\sigma_{i}(v)$ are the threshold frequency and cross section for photoionization of species $i$, given in Osterbrock (1989; see eq. [2.31]) for $\mathrm{H} \mathrm{I}, \mathrm{He} \mathrm{I}$, and $\mathrm{He} \mathrm{II}$ ]. For the external photons the $\mathrm{UV}$ intensity is $I_{v, 0} e^{-\tau_{v}}$, with the boundary intensity $I_{v, 0}=I_{v_{\mathrm{L}}, 0}\left(v / v_{\mathrm{L}}\right)^{-\alpha}$ as before, and $\tau_{v}$ approximated as $\tau_{v_{\mathrm{L}}}\left(v / v_{\mathrm{L}}\right)^{-3}$. Since $\sigma_{i}(v)$ has the simple form of a sum of two power laws, the frequency integral in $\Gamma_{\gamma i}$ can be done analytically, and only the angular integration is computed numerically (see the similar but simpler calculation of Tajiri \& Umemura 1998). There is an additional contribution to photoionization for $\mathrm{H} \mathrm{I}$ only, from the emitted photons just above $v=v_{\mathrm{L}}$, given by $\int_{0}^{4 \pi} d \Omega \sigma_{i}\left(v_{\mathrm{L}}\right) F_{1}$. The photoheating rate per unit volume is $n_{i} \epsilon_{i}$, where $n_{i}$ is the number density of species $i$ and

$$
\epsilon_{i}=\int_{0}^{4 \pi} d \Omega \int_{v_{i}}^{\infty} \frac{I_{v}}{h v} \sigma_{i}(v)\left(h v-h v_{\mathrm{L}}\right) d v \operatorname{ergs~s}^{-1} .
$$

The rate for the external UV radiation is calculated for each atomic species similarly to the calculation of $\Gamma_{\gamma i}$. The emitted photons contribute to $\epsilon_{\mathrm{H}}$ an extra amount of $\int_{0}^{4 \pi} d \Omega \sigma_{i}\left(v_{\mathrm{L}}\right) F_{2}$.

Abel, T., \& Mo, H. J. 1998, ApJ, 494, L151

Abel, T., Norman, M. L., \& Madau, P. 1999, ApJ, 531, in press

Babul, A., \& Rees, M. 1992, MNRAS, 253, 31

Barnes, J. E., \& Hernquist, L. 1992, Nature, 360, 715

Bertoldi, F. 1989, ApJ, 346, 735

Bertoldi, F., \& McKee, C. F. 1990, ApJ, 354, 529

\section{REFERENCES}

Black, J. H. 1981, MNRAS, 197, 553

Bond, J. R., Szalay, A. S., \& Silk, J. 1988, ApJ, 324, 627

Bryan, G. L., Machacek, M., Anninos, P., \& Norman, M. L. 1998, ApJ, 517, 13

Bryan, G., \& Norman, M. 1998, ApJ, 495, 80

Burkert, A. 1995, ApJ, 447, L25 
Crotts, A. P. S, \& Fang, Y. 1998, ApJ, 502, 16

Duc, P.-A., \& Mirabel, I. F. 1997, in Proc. IAU Symp. 187, Cosmic Chemical Evolution, ed. J. W. Truran \& K. Nomoto (Dordrecht: Kluwer), in press

Dekel, A., \& Silk, J. 1986, ApJ, 303, 39

Dey, A., Spinrad, H., Stern, D., Graham, J. R., \& Chaffee, F. H. 1998, ApJ, 498, L93

Donahue, M., \& Shull, J. M. 1987, ApJ, 323, L13

Elmegreen, B. G., Kaufman, M., \& Thomasson, M. 1993, ApJ, 412, 90

Efstathiou, G. 1992, MNRAS, 256, 43

Fall, S. M., \& Rees, M. J. 1985, ApJ, 298, 18

Ferland, G. J., Peterson, B. M., Horne, K., Welsh, W. F., \& Nahar, S. N. 1992, ApJ, 387, 95

Franco, J., Ferrara, A., Roczyska, M., Tenorio-Tagle, G., \& Cox, D. P. 1993, ApJ, 407, 100

Fusi-Pecci, F., Ballazzini, M., Cacciari, C., \& Ferraro, F. R. 1995, AJ, 100, 1664

Garnavich, P. M., et al. 1998, ApJ, 509, 74

Gnedin, N. Y., \& Ostriker, J. P. 1997, ApJ, 486, 581

Grebel, E. 1998, in IAU Symp. 192, The Stellar Content of Local Group Galaxies, ed. P. Whitelock \& R. Cannon (San Francisco: ASP)

Haiman, Z., \& Loeb, A. 1998, ApJ, 503, 505 1999, ApJ, 519, 479

1998c, in AIP Conf. Proc. 470, After the Dark Ages: When Galaxies Were Young (the Universe at $2<z<5$ ), ed. S. Holt \& E. Smith (Woodbury: AIP),

Haiman, Z., Rees, M., \& Loeb, A. 1996a, ApJ, 476, 458 (erratum 484, 985 [1997])

Haiman, Z., Thoul, A. A., \& Loeb, A. 1996b, ApJ, 464, 523

Haehnelt, M. G., \& Steinmetz, M. 1998, MNRAS, 298, 21

Heggie, D. C., \& Hut, P. 1995, in IAU Symp. 174, Dynamical Evolution of Star Clusters-Confrontation of Theory and Observations, ed. P. Hut \& J. Makino (Dordrecht: Kluwer), 303

Hu, E. M., Cowie, L. L., \& McMahon, R. G. 1998, ApJ, 502, L99

Katz, N., Weinberg, D. H., \& Hernquist, L. 1996, ApJS, 105, 19

Kepner, J. V., Babul, A., \& Spergel, D. N. 1997, ApJ, 487, 61

Klypin, A. A., Kravtsov, A. V., Valenzuela, O., \& Prada, F. 1999, ApJ, 522, 82

Kravtsov, A. V., Klypin, A. A., Bullock, J. S., \& Primack, J. R. 1998, ApJ, 502,48

Kroupa, P. 1997, NewA, 2, 139

Lacey, C. G., \& Cole, S. M. 1993, MNRAS, 262, 627

Larson, R. B. 1974, MNRAS, 271, 676L

Lynden-Bell, D. \& Lynden-Bell, R. M. 1995, MNRAS, 275, 429

Mac Low, M., \& Ferrara, A. 1999, ApJ, 513, 142

Majewski, S. R. 1994, ApJ, 431, L17

Mateo, M. 1998, ARA\&A, 36, 435
Miller, B. W., Whitmore, B. C., Schweizer, F., \& Fall, S. M. 1997, AJ, 114, 2381

Miralda-Escudé, J. 1998, ApJ, 501, 15

Miralda-Escude, J., Haehnelt, M., \& Rees, M. J. 1999, ApJ, submitted (astro-ph/9812306)

Miralda-Escude, J., \& Rees, M. J. 1998, ApJ, 497, 21

Moore, B. 1996, ApJ, 461, L13

Moore, B., Governato, F., Quinn, T., Stadel, J., \& Lake, G. 1998, ApJ, 499, L5

Navarro, J. F., Frenk, C. S., \& White, S. D. M. 1997, ApJ, 490, 493 (NFW)

Navarro, J. F., \& Steinmetz, M. 1997, ApJ, 478, 13

Olszewski, E. W. 1998, in Galactic Halos: A UC Santa Cruz Workshop, ed. D. Zaritski (San Francisco: ASP)

Osterbrock, D. E. 1989, Astrophysics of Gaseous Nebulae and Active Galactic Nuclei (Mill Valley: University Science Books)

Peebles, P. J. E. 1993, Principles of Physical Cosmology (Princeton: Princeton Univ. Press)

Peebles, P. J. E., \& Dicke, R. H. 1968, ApJ, 154, 891

Pen, U.-L. 1998, ApJ, 498, 60

Petry, C. E., Impey, C. D., \& Foltz, C. B. 1998, ApJ, 494, 60

Press, W. H., \& Schechter, P. 1974, ApJ, 187, 425

Quinn, T., Katz, N., \& Efstathiou, G. 1996, MNRAS, 278, L49

Razoumov, A., \& Scott, D. 1999, MNRAS, submitted (astro-ph/9810425)

Rees, M. J. 1986, MNRAS, 218, 25

Shapiro, P. R., \& Kang, H. 1987, ApJ, 318, 32

Shapiro, P. R, Raga, A. C. \& Mellema, G. 1998, in Molecular Hydrogen in the Early Universe, Memorie Della Societa Astronomica Italiana, Vol. 69, ed. E. Corbelli, D. Galli, \& F. Palla (Florence: Soc. Astron. Italiana), 463

Songaila, A., \& Cowie, L. L. 1996, AJ, 112, 335

Spinrad, H., Stern, D., Bunker, A., Dey, A., Lanzetta, K., Yahil, A., Pascarelle, S., \& Fernández-Soto, A. 1998, AJ, 116, 2617

Spitzer, L., Jr. 1978, Physical Processes in the Interstellar Medium (New York: Wiley)

Spitzer, L., Jr., \& Hart, M. H. 1971, ApJ, 166, 483

Stecher, T. P., \& Williams, D. A. 1967, ApJ, 149, L29

Tajiri, Y., \& Umemura, M. 1998, ApJ, 502, 59

Thoul, A. A., \& Weinberg, D. H. 1996, ApJ, 465, 608

Tytler, D., Fan, X.-M., Burles, S., Cottrell, L., Davis, C., Kirkman, D., \& Zuo, L. 1995, in QSO Absorption Lines, ed. G. Meylan (Berlin: Springer), 289

Unavane, M., Wyse, R. F. G., \& Gilmore, G. 1996, MNRAS, 278, 727

Vader, J. P. 1986, ApJ, 305, 669

. 1987, ApJ, 317, 128

Verner, D. A., \& Ferland, G. J. 1996, ApJS, 103, 467

Voronov, G. S. 1997, At. Data Nucl. Data Tables, 65, 1

Weinberg, D. H., Hernquist, L., \& Katz, N. 1997, ApJ, 477, 8 